\title{
STATISTICAL ASSEMBLIES WITH FORM ERRORS - A 2D EXAMPLE
}

\author{
Pierre-Antoine Adragna, Hugues Favrelière, Serge Samper, Maurice Pillet \\ Symme Lab. Université de Savoie, 5 chemin de Bellevue 74940 Annecy le Vieux \\ \{pierre-antoine.adragna, hugues.favreliere, serge.samper, maurice.pillet\}@univ-savoie.fr
}

\begin{abstract}
When dealing with precision in tolerancing of assembly systems, the modelling complexity of the mechanism increases. At first, one can distinguish the 1D tolerancing approach that only concerns variations of dimension. Then, several models are defined to set $3 \mathrm{D}$ tolerances, considering that the form error is negligible compared to the orientational and translational variations. Finally, some approaches are proposed to take into account the form variations in the tolerancing of mechanisms. However, some modelling approaches considers the form error as a tolerance zone to add to the $3 \mathrm{D}$ tolerances as defined by Rule\# 1 of the ASME standard, or ISO 8015. This paper proposes another point of view, considering the positioning of parts through contact points of their rigid deviation shapes under a defined assembly force and set-up. Rather than considering the positioning of a single part, here is proposed an approach of batch parts assembly by a statistical description of shapes. The result of the method is a statistical positioning error of one part on the other considering the form deviations of parts.
\end{abstract}

Keywords 3D assembly, form errors, positioning force, statistical assembly

\section{Introduction}

The tolerancing of an assembly system is based on a model of the mechanism. Depending on the complexity of this model large errors can be obtained on the parts positioning. The most simple modelling that can be identified only considers the dimensional variations as presented by Graves [1] for example. More complex models of the mechanism are developed to exploit the 3D tolerance zone. Many methods are proposed such as the vector chain of Chase [2], the T-Maps of Davidson and his team [3], the small displacement torsor (SDT) of Bourdet [4], and the clearance and deviation domains of Giordano and his team [5]. But as the precision of the mechanism increases, the form deviation of shapes has to be considered. Ameta [6] proposes a model in the T-Maps space to study the influence of the form deviation based on the tolerance zone, but only one form deviation is as yet considered. Radouani [7] presents an experimental study of the positioning of one part on another regarding the size of their form deviations. Neville [8] gives an 
algorithm to determine the positioning error of a butting assembly of two components considering their form roughness and dimensional error with or without an auxiliary surface. Our solution [9] is based on the identification of the contact points of the two faces given by a mechanism defined by external face data and a positioning torsor expressed by a $F$ force located on an axis. Our method allows the prediction of the positioning error into the SDT space.

Figure 1 below shows the chosen example for this paper. We reduce the study to a two dimensional profile assembly. The two parts are linked by the " $A_{i}$ " joint and the functional requirement " $\mathrm{Cf}$ " is given by the clearance between the " $B_{i}$ " faces. $B_{i}$ faces are supposed to be perfect in this example. The point of interest is their relative displacements according to the assembly variations due to the $A_{i}$ faces.

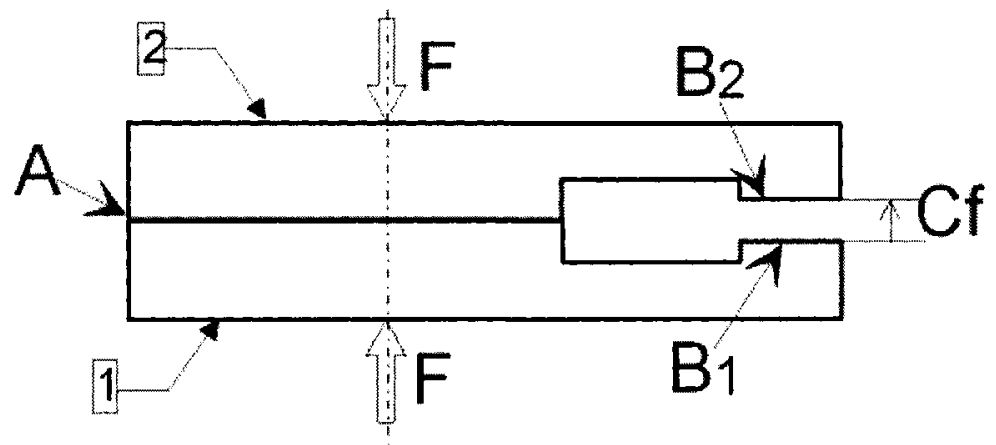

Fig. 1. 2D assembly and its functional requirement

\section{The proposed method and background}

\subsection{A single assembly}

Our approach consists of identifying all the possible contact points of the two faces regarding their form deviations and a given pre-positioning mechanism. The final contact points that define the stable positioning are identified by a positioning force.

The first step of the method is the identification of the possible contact points. To do so, the difference surface is introduced. This surface corresponds to the difference of the form deviations, and represents point to point distances. This difference surface can be found by a point to point distance computation, but the modal analysis of form deviation is recommended. Figure 2 presents the difference surface computed point to point. One can observe the roughness of the measured shapes that is filtered by the use of the modal characterisation.

The second step of our approach is the identification of all the possible contact points between the two parts surfaces. The proposed solution is the use of the 
convex hull that identifies contact faces, hence the contact points. Figure 2 shows the convex surface of the difference surface and the identified potential contact points.
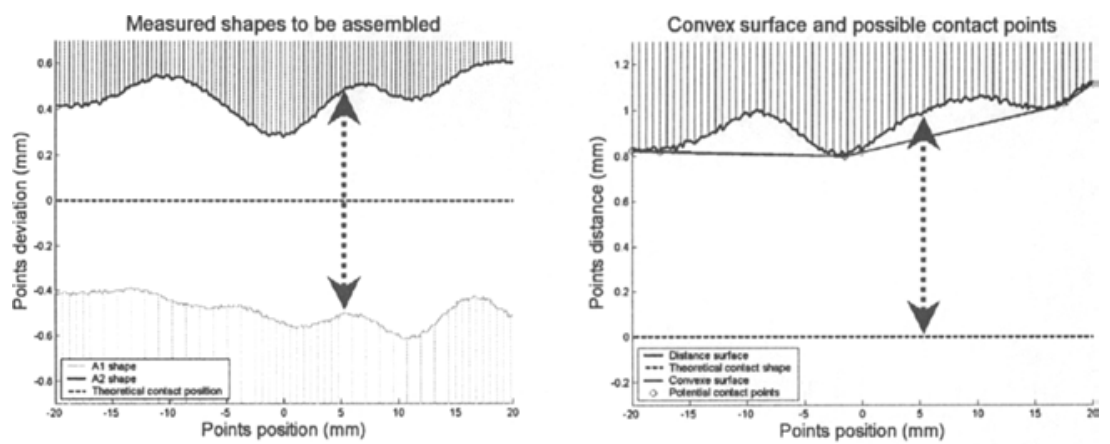

Fig. 2 a) parts form deviations $A_{l}$ and $A_{2}, b$ ) relative distance surface
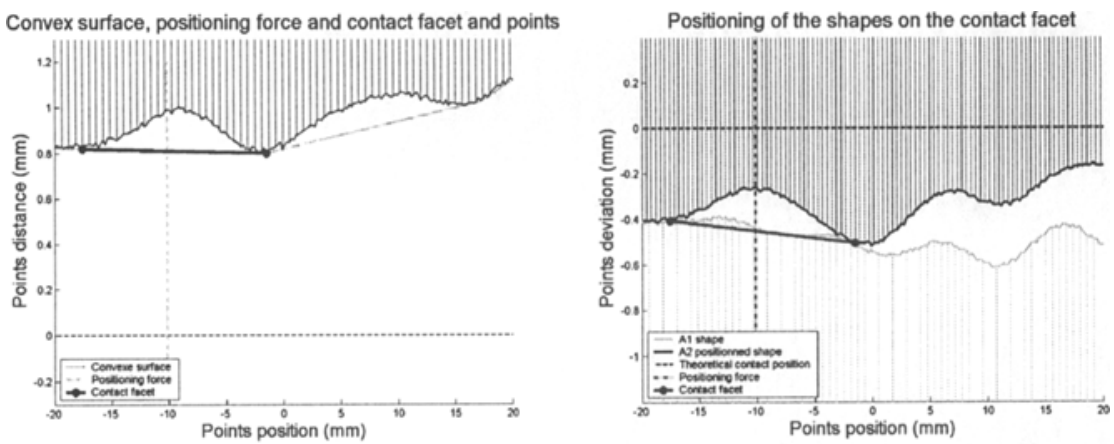

Fig. 3 a) Convex surface, $b$ ) positioning deviation according to the positioning force location

The third and last step is the identification of the contact facet, giving the contact points. A solution is the use of a positioning force, whose location of the direction identifies the contact facet. As a rigid model is considered, the force intensity is not used. The identified contact facet corresponds to the opposite of the positioning error. Figure 3 shows the identified contact facet for a given position force location and the parts assembly corresponding. The positioning deviation corresponds to the deviation of the identified facet to the theoretical contact position. 


\subsection{Modal analysis}

Introduced by the team of Samper [10-11], the modal analysis of form deviations is a generic approach to building a basis of form errors for any shapes. Hence any form deviation can be analysed in this modal basis and described as a set of coefficient as for the Fourier transform. The modal analysis method is based on the modal shapes of vibrations for the ideal geometry. These shapes have interesting properties such as:

- it is a vectorial basis of form errors,

- modals shapes are naturally sorted by growing complexity,

- modal shapes are easily calculated for any kind of geometry (use of the Finite Element Model to solve complex shapes)

The method is based on the space solution of the natural vibrations of forms. The following equation 1 is the d'Alembert equation of vibrations.

$\square(\mathrm{U})=0 \Leftrightarrow \nabla^{2} \mathrm{U}=\frac{1}{c^{2}} \frac{\partial^{2} U}{\partial t^{2}}$
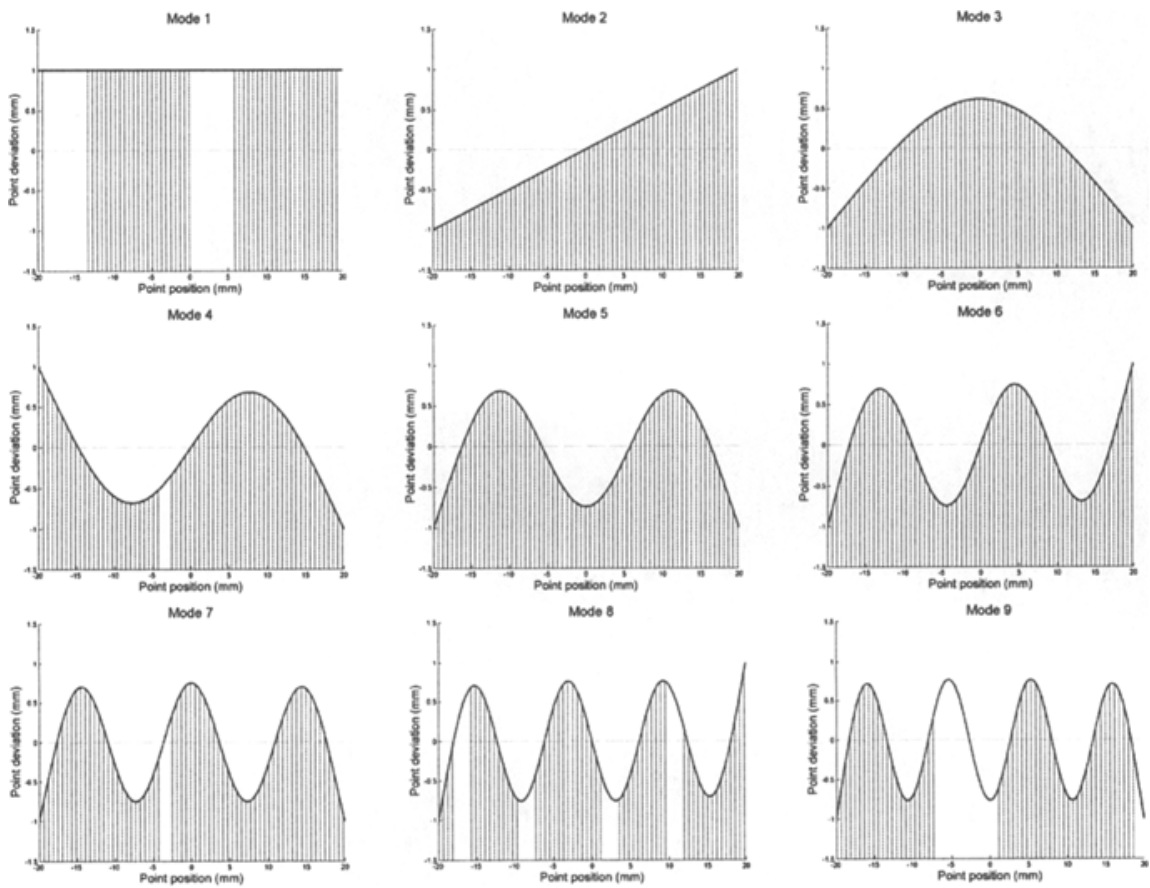

Fig. 4. First nine modal basis of our $A_{i}$ contact $2 \mathrm{D}$ profiles 
This equation can be solved by an analytical way for simple cases or by a numerical way for most of cases. The obtained vibration modes are considered as form deviations $\Phi_{i}$. These $\Phi_{i}$ shapes are modified to obtain a orthogonal basis, and in order to have a metric meaning of the modal coefficients, the modal shapes amplitude are set to the unit [11]. Figure 4 shows the first nine form errors of the modal basis of our case of application.

\subsubsection{Analysis of a single shape}

The first interest of the use of the form deviation modal analysis is the filtering of the form roughness, and the reduction of the number of characteristics that describing the form deviation. The following figure 5 shows the modal characterisation of an error shape, called the modal signature of the form deviation in the modal basis, and the recomposed form corresponding to the filtered shape. The result of the modal characterisation of a measured form deviation $V$ on the $B$ modal basis is the modal signature $\Lambda$. This $\Lambda$ modal signature is composed of the $\lambda_{i}$ modal coefficients calculated by the following relation.

$\lambda_{\mathrm{i}}=$ B $. \Phi_{\mathrm{i}} /\left\|\Phi_{\mathrm{i}}\right\|^{2}$

The recomposed shape with the rigid modes (translation and rotation) corresponds to the rigid shape and is equivalent to the Least Square associated shape. The recomposed shape $R$ is obtained by the following relation:

$\mathrm{R}=\Lambda \cdot \mathrm{B}$
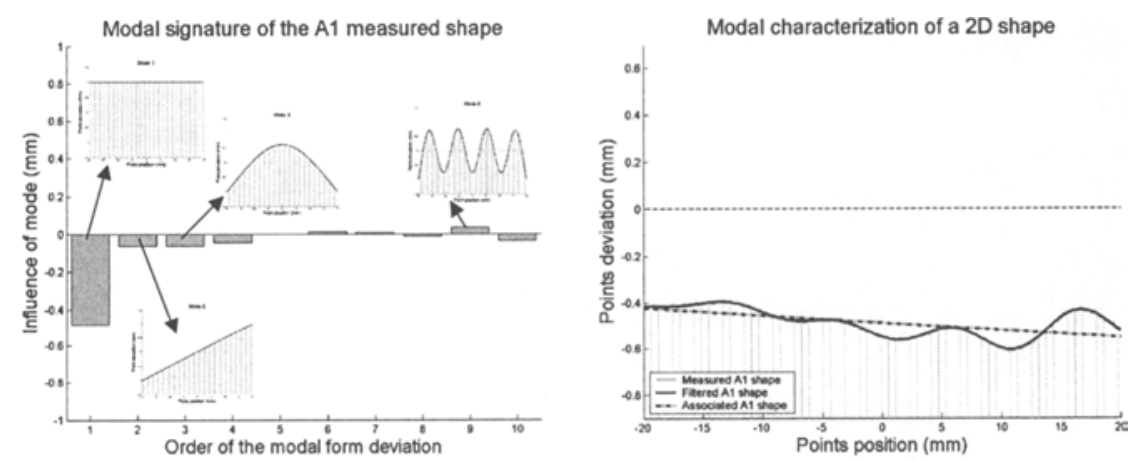

Fig. 5 a) modal signature, b) recomposed shape 


\subsubsection{Simulation of shapes}

The modal characterisation of form errors can be used to create simulated shapes. A random draw of the modal coefficients creates a random shape which form complexity depends on the number of considered modes. Based on the observation of shapes analyses, we consider that the amplitude of the modal coefficients is given by the following law:

$A(i)=A_{0} / i$

Where $A_{0}$ is initial amplitude, $i$ is the order of the modal coefficient and $A(i)$ is the maximum amplitude of the $i^{\text {th }}$ modal coefficient. The following Figure 6 shows the amplitude law of the coefficients and a random draw of a modal signature.
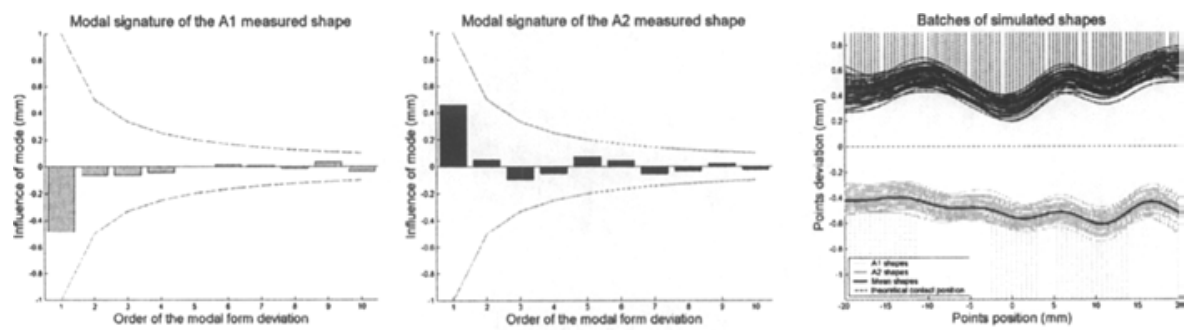

Fig. $6 a$ and $b$ ) random modal signatures, c) batches of simulated form deviations

The simulation of a batch of form deviations can be obtained from an initial modal signature, on which are added random modal variations. The amplitude of these modal form variations has the same type of law as the initial form deviation. Another random variation can be added on the on the recomposed shapes that can represent roughness or measurement error. A set of 50 simulated shapes are presented in figure 6.

\subsubsection{Statistical analysis of a set of shapes}

The second interest of the modal analysis method is the statistical description of a set of deviation shapes. Presented in CIRP-CAT 2007 [12-13], the modal analysis is extended to the qualification of a batch of form deviations. From the batch of modal signatures $\Lambda_{i}$ (characterizations of the shapes), one is able to compute the mean modal signature $\mu_{\Lambda i}$ and the covariance matrix $\Sigma_{\Lambda i}$, whose root of the diagonal represents the standard deviation modal signature $\sigma_{A i}$. It is also possible to have a geometrical representation of this statistical qualification. The following relation links the mean form deviation of the batch to the mean modal signature:

$\mu_{V}=\mathrm{B} \cdot \mu_{\Lambda}$ 
The following relation links the covariance matrix of the form deviations to the covariance matrix of the modal signatures:

$$
\Sigma_{\mathrm{V}}=\mathrm{B} \cdot \Sigma_{\Lambda} \cdot \mathrm{B}^{\prime}
$$

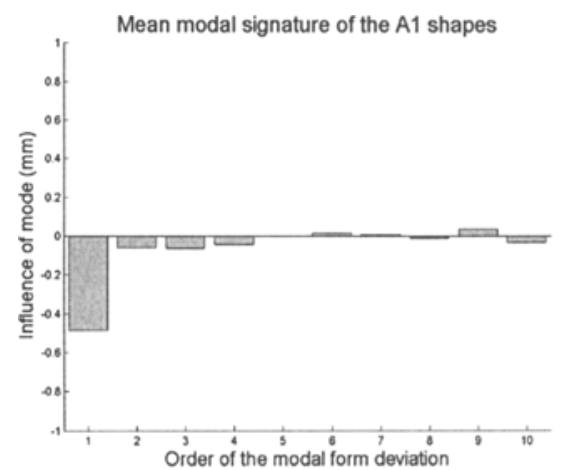

Fig. 7 a) mean modal signature, b) covariance matrix of the modal coefficients

\section{The statistical assembly of form deviations}

This section details our proposed method to deal with the assembly of a batch of components. A first solution is to use the approach of the first part to compute single assembly. This method will be used to confirm the following proposition. The idea of the statistical assembly is to find the mean positioning of the batch, and then the standard deviation around this mean positioning.

\subsection{Mean positioning and contact points}

The first step of our approach is to determine the mean positioning and the contact points associated. These mean contact points are used to determine the mean positioning and its standard deviation linked to the means and standard deviations of the parts form deviations.

This first step consists of solving the positioning of the two mean shapes of the two parts batches. This is achieved by the previous approach of assembling single parts, except that parts are mean forms. One hence obtains the mean positioning error of the parts batches, and more important is the identification of the contact points. 

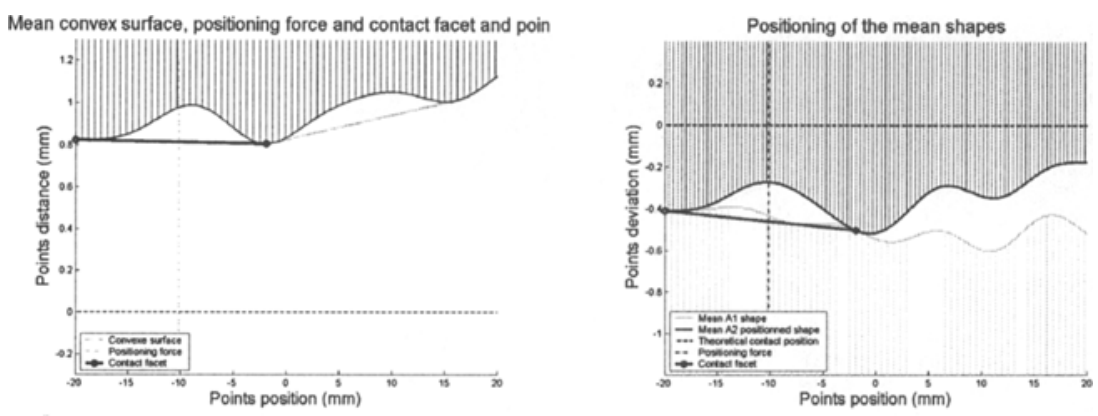

Fig. 8 a) identified mean contact facet, b) mean positioning

\subsection{Standard deviation of the positioning error}

Thanks to the identification of the contact points, standard deviation surfaces of the parts form errors can be linked to the standard deviation of the positioning error. The solution we choose is to consider the covariance matrix of the contact points, given by the covariance of the measured form deviations or by the modal characterisation. As parts are independent, the covariance matrix of the positioning error is the sum of the covariance matrices of the parts form deviations. In our case, the two contact points are identified as the $1^{\text {st }}$ and the $91^{\text {st }}$ measured points. Hence, it is possible to predict the positioning covariance matrix from the covariance matrix of the contact points.

The following Figure 9 shows a particular positioning of shapes from the two batches. It can be observed that the contact points of this particular assembly are not identical to the contact points of the mean shapes positioning.
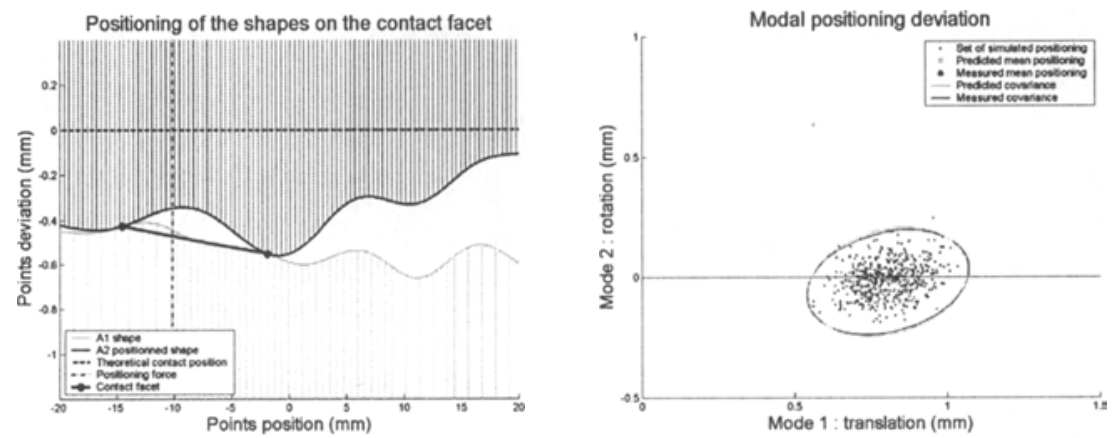

Fig. 9 a) a particular assembly from the batches, $b$ ) the positioning results and prediction 
However, the positioning result of the batches is showed by the blue dots in Figure 9. The red dot corresponds to the mean positioning error of the results and the red ellipse corresponds to 3 times the covariance of the results. The prediction of our approach is represented by the green parts. The green dot corresponds to the predicted mean positioning error, and the green ellipse is 3 times the predicted positioning covariance. It can be observed on this simulation that the predicted results are similar to the simulated ones.

Another simulation with a larger shape variations and another location of the positioning force is showed in the next figure.
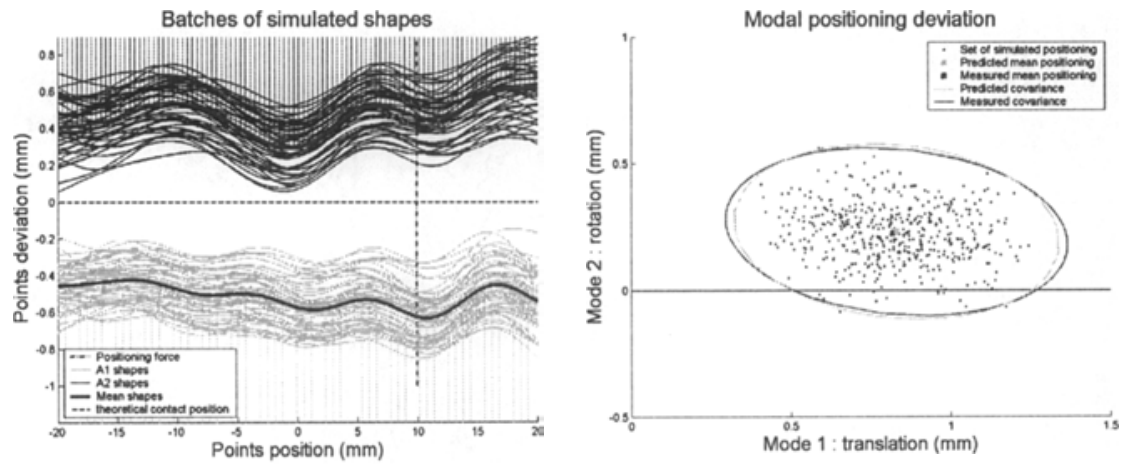

Fig. 10 a) batches of shapes, $b$ ) associated positioning results and prediction

With the new simulation, as the shape variation is more important, the identification of the contact points is less accurate. However the predicted result is close to the statistical characterisation of the simulations.

The results are presented in the modal coordinates that can be transcribed into a small displacement torsor as follows. Thus, the positioning deviation of the A2 shape can be transferred to the B2 shape to check the respect of the functional requirement " $\mathrm{Cf}$ ".

\section{Conclusion}

Surface assembly considering the form error is treated by the use of the modal parameterisation. This method allows us to describe form errors of all geometries in an exhaustive way. It also provides a filtering of the form deviation to keep influent modes and reject roughness.

The problem of non determination of the assembly is solved using the force torsor. Its location allows us to find the contact points of both faces by the use of the distance surface. The characterisation of the contact facet gives positioning deviation of surface 2 on surface 1 . 
The proposed approach to deal with statistical assemblies of form errors is interesting in the way that form deviation of shapes are transcribed into positioning error. This positioning deviation is expressed in the modal space in our case, but can also be described in small displacement torsor of T-Map.

The next step of our approach is the simulations on more complex cases (more variation, and 3D components), and the confrontation of our theory to measured assemblies.

\section{References}

1. S. Graves, Tolerance Analysis Formula Tailored to Your Organization, Journal of Quality Technology, Vol 33, No 3, July 2001

2. K.W. Chase, Tolerance Analysis of a 2D and 3D assemblies, ADCATS Report, No $94-4,1999$

3. J.K. Davidson, J.J, Shah, Geometric Tolerances : A New Application for Line Geometry and Screws, ImechE Journal of Mechanical Engineering Science, Vol 216, Part C, 2002 pp. 95-104

4. P. Bourdet, L. Mathieu, C. Lartigue, A. Ballu, The Concept of Small Displacement Torsor in Metrology, Proceedings of the International Euroconference, Advanced Mathematical Tools in Metrology, Oxford, September 27-30 1995

5. M. Giordano, B. Kataya, E. Pairel, Tolerance Analysis and Synthesis by Means of Clearance and Deviation Spaces, Proceedings of the $7^{\text {th }}$ CIRP International Seminar on Computer Aided Tolerancing, 2001, pp. 345-354

6. G. Ameta, J.K. Davidson, J. Shah, Influence of Form Frequency Distribution for a 1D Clearance Wich is Generated from Tolerance-Maps, Proceedings of the $10^{\text {th }}$ CIRP Conference on Computer Aided Tolerancing, Specification and Verification for Assemblies, Erlangen Germany, March 21-23 2007

7. M. Radouani, B. Anselmetti, Contribution à la Validation du Modèle des Chaînes de Cotes - Etudes Expérimentale du Comportement de la Liaison Plan sur Plan, Congrès International Conception et Production Intégrées, CPI 2003, Meknès Moroco, October 22-24 2003

8. K.S.L. Neville, Y. Grace, The modeling and analysis of butting assembly in the presence of workpiece surface roughness part dimensional error, International Journal of Advanced Manufacturing Technologies, Vol. 31, 2006, pp. 528-538

9. P.-A. Adragna, S. Samper, H. Favrelière, M. Pillet, Analyse d'un assemblage avec prise en compte des défauts de forme, Congrès International Conception et Production Intégrées, CPI 2007, Rabat Morocco, October 22-24 2007

10. F. Formosa, S. Samper, I. Perpoli, Modal expression of form defects, in Models for Computer Aided Tolerancing in Design and Manufacturing, Springer series 2007, pp. 13-22

11. P.-A. Adragna, S. Samper, M. Pillet, Analysis of Shape Deviations of Measured Geometries with a Modal Basis, Journal of Machine Engineering: Manufacturing Accuracy Increasing Problems - Optimization, Vol. 6, No 1, 2006, pp. 95-102 
12. P.-A. Adragna, S. Samper, M. Pillet, Inertial tolerancing applied to 3D and form tolerancing with the modal analysis, Proceedings of the $10^{\text {th }} \mathrm{CIRP}$ Conference on Computer Aided Tolerancing, Specification and Verification for Assemblies, Erlangen Germany, March 21-23 2007

13.H. Favrelière, S. Samper, P.-A. Adragna, M. Giordano, 3D statistical analysis and representation of form error by a modal approach, Proceedings of the $10^{\text {th }}$ CIRP Conference on Computer Aided Tolerancing, Specification and Verification for Assemblies, Erlangen Germany, March 21-23 2007 